\title{
The Quantization and Renormalization of Gravitational Field
}

\author{
Bin Liang ${ }^{1}$ \\ ${ }^{1}$ College of Science, Chongqing University of Posts and Telecommunication, Chongqing 400065, China \\ Correspondence: Bin Liang, College of Science, Chongqing University of Posts and Telecommunication, \\ Chongqing 400065, China. E-mail: liangbin@cqupt.edu.cn
}

Received: March 4, 2015 Accepted: March 27, 2016 Online Published: March 31, 2016

doi:10.5539/apr.v8n2p93 URL: http://dx.doi.org/10.5539/apr.v8n2p93

\begin{abstract}
This paper analyses the root cause why there is not any self-consistent quantization scheme of gravity yet from Einstein's theory of gravity, proves the gravitational field is a gauge field and gives the quantization and renormalization scheme of the gravitational field.
\end{abstract}

Keywords: Einstein's theory of gravity, gauge field, quantization and renormalization, dimension regularization

\section{Introduction}

The core idea of Einstein's theory of gravity is that the gravitational effect results in the curve of space-time, and the motion of body in gravitational field is equivalent to the motion in the curved space-time (Einstein, 1916). Einstein and others had obtained many important results consistent with the observation on the basis of the principle above. However, there is an important problem troubling physicists for a long time, it is the quantization of gravitational field. It is impossible to unify the gauge field theories to describe the four interactions if the problem is not solved.

We know that the quantization of field must be carried out in a certain background space-time, and the gravitational field is not an exception. If we take the curved space-time as the background space-time for the quantization of gravitational field, since the covariant differential of the metric tensor $g_{\mu \nu}$ equals zero:

$$
g_{\mu v ; \lambda}=0,
$$

the quantization of gravitational field is impossible, because no matter what the quantization method, canonical quantization or other quantization methods, it is inseparable from a physical reasonable Lagrange density, and a reasonable Lagrange density must contain the physical quantities and their derivatives (Weinberg, 1972). But it is impossible to find the Lagrange density $L\left(g_{\mu v}, g_{\mu v ; \lambda}\right)$ containing the covariant differential $g_{\mu v ; \lambda}$ in the curved space-time due to the equation above, so the quantization of gravitational field is impossible in the curved space-time. In other words, we cannot take the curved space-time both as the quantized object and the background space-time. Otherwise, we would get into paradox in one way or another. This is the root cause why we do not have a self-consistent quantization scheme of the gravitational field yet.

On the other hand, a local inertial system can be set up at any point in the curved space-time, in which the special relativity is established according to the equivalence principle. In fact, the discussion about the localized field theory in the local flat space-time is only natural. For example, people discussed the gravitational waves on the condition of weak field approximation and had established the tetrad formation in the local flat space-time (Weinberg, 1972).

According to the above analysis, the gravitational field should and could be quantized in the local flat space-time. We will see that the gravitational field could be very naturally quantized and renormalized from the Lagrange density of gravitational field in the local flat space-time.

\section{The Gravitational Potential: From Tensor to Vector}

The Lagrange density of gravitational field used to derive the Einstein's equation could be written as (Weinberg, 1972)

$$
L_{g}=-\sqrt{g} R
$$


where $g=-\operatorname{Det}\left(g_{\mu \nu}\right)$, the scalar curvature is

$$
R=g^{\lambda v} g^{\mu k} R_{\lambda \mu v k},
$$

the tensor curvature is

$$
R_{\lambda \mu \nu k}=\frac{1}{2}\left(g_{\lambda v, \mu k}+g_{\mu k, \lambda v}-g_{\mu v, \lambda k}-g_{\lambda k, \mu v}\right)+g_{\eta \sigma}\left(\Gamma_{\lambda v}^{\eta} \Gamma_{\mu k}^{\sigma}-\Gamma_{\lambda k}^{\eta} \Gamma_{\mu \nu}^{\sigma}\right),
$$

and the connection is

$$
\Gamma_{\lambda v}^{\eta}=\frac{1}{2} g^{\eta \rho}\left(g_{\lambda \rho, v}+g_{\rho v, \lambda}-g_{\nu \lambda, \rho}\right) .
$$

The quantities above are the functional of coordinate $x$ in the local flat space-time.

The canonical physical quantity conjugated with the gravitational potential $g_{\alpha \beta}$ is defined as

$$
\pi^{\alpha \beta}=\frac{\partial L_{g}}{\partial g_{\alpha \beta, 4}} .
$$

Substitute the previous several equations into the equation above, we obtain

$$
\pi^{\alpha \beta}=-\sqrt{g} g_{\eta \sigma} g^{\lambda v} g^{\mu k} \frac{\partial}{\partial g_{\alpha \beta, 4}}\left(\Gamma_{\lambda v}^{\eta} \Gamma_{\mu k}^{\sigma}-\Gamma_{\lambda k}^{\eta} \Gamma_{\mu \nu}^{\sigma}\right) .
$$

Take the harmonic coordinates: $\Gamma^{\eta}=g^{\lambda v} \Gamma_{\lambda v}^{\eta}=0$, there is

$$
\begin{aligned}
\pi^{\alpha \beta} & =\sqrt{g} g_{\eta \sigma} g^{\lambda v} g^{\mu k} \frac{\partial}{\partial g_{\alpha \beta, 4}}\left(\Gamma_{\lambda k}^{\eta} \Gamma_{\mu v}^{\sigma}\right) \\
& =2 \sqrt{g} g_{\eta \sigma} g^{\lambda v} g^{\mu k} \Gamma_{\lambda k}^{\eta} \frac{\partial \Gamma_{\mu \nu}^{\sigma}}{\partial g_{\alpha \beta, 4}} \\
& =\sqrt{g} g^{\lambda v} g^{\mu k}\left(g_{\lambda \sigma, k}+g_{\sigma k, \lambda}-g_{k \lambda, \sigma}\right) \frac{\partial \Gamma_{\mu \nu}^{\sigma}}{\partial g_{\alpha \beta, 4}} \\
& =\frac{1}{2} \sqrt{g}\left(-3 g^{4 \lambda} g_{, \lambda}^{\alpha \beta}+g^{\beta k} g_{, k}^{\alpha 4}+g^{\alpha \lambda} g_{, \lambda}^{\beta 4}\right) .
\end{aligned}
$$

There is $\pi^{\alpha \beta}=\pi^{\beta \alpha}$ obviously.

Image the metric $g^{\alpha \beta}$ is orthogonal for space-time axes: $g^{\alpha \beta}=g^{\alpha} \delta_{\alpha \beta}$ (the repeat subscripts are not summed here), then there are only four independent components of $g^{\alpha \beta}: g^{i}(i=1,2,3)$ and $g^{4}$, they form a 4-vector. So there is

$$
\begin{aligned}
& \pi^{i i}=-\frac{3}{2} \sqrt{g} g^{4} g_{, 4}^{i}, \\
& \pi^{44}=-\frac{1}{2} \sqrt{g} g^{4} g_{, 4}^{4}, \\
& \pi^{4 i}=\frac{1}{2} \sqrt{g} g^{i} g_{, i}^{4},
\end{aligned}
$$

and the rest components of $\pi^{\alpha \beta}$ equal to zero.

Assume the 4-gravitational potential satisfies the Lorentz condition: 


$$
g_{, \mu}^{\mu}=0 \text {. }
$$

Notice that the components of $\pi^{\alpha \beta}$ in the equation (9) have nothing to do with $g_{, j}^{i}$, take $g_{, j}^{i}=0$, then $g_{, 4}^{4}=0$ and obtain

$$
\begin{aligned}
\pi^{i i} & =-\frac{3}{2} \sqrt{g} g^{4} g_{, 4}^{i}, \\
\pi^{4 i} & =\frac{1}{2} \sqrt{g} g^{i} g_{, i}^{4} .
\end{aligned}
$$

This is the gravitational field intensity.

In the case of static weak field there are

$$
g^{i}=1-h^{i}, \quad g^{4}=-1-h^{4}, \quad h^{4}=2 \phi .
$$

So there is

$$
\boldsymbol{E} \equiv \pi^{4 i}=-\nabla \phi .
$$

This is the intensity of Newton's gravitational field, where Newton's gravitational potential is

$$
\phi(r)=-\frac{G M}{r} \text {. }
$$

\section{The Classic Gravitational Field}

As mentioned previously, the gravitational potential is a 4-vector in the local flat space-time on the condition of harmonic coordinates and orthogonal space-time axes. So, the gravitational potential could be written as $A_{\mu}=$ $\left(\boldsymbol{A}, A_{4}=i U / c\right)$ and the source material flow density as $j_{\mu}=\left(\boldsymbol{j}, j_{4}=i c \rho\right), \quad c$ is the speed of gravitational wave. We will show the speed $c$ equals the speed of light in a vacuum later.

Since the transmission speed of gravity is limited, the gravitational potential should be retarded. Assume the source material flow density $j_{\mu}\left(x^{\prime}\right)=j_{\mu}\left(t^{\prime}\right) \delta\left(\boldsymbol{x}^{\prime}\right)$ in the local Minkowski space $x\left(\boldsymbol{x}, x_{4}=i c t\right)$, so the scalar potential is

$$
U(x)=-G \frac{\rho\left(t^{\prime}\right)}{r} \delta\left(x^{\prime}\right)
$$

the vector potential is

$$
\boldsymbol{A}(x)=-\frac{\eta}{r} \boldsymbol{j}\left(t^{\prime}\right) \delta\left(\boldsymbol{x}^{\prime}\right)
$$

where $r=\left|\boldsymbol{x}-\boldsymbol{x}^{\prime}\right|, t^{\prime}=t-\frac{r}{c}$, and $\eta$ is a constant. Obviously, the material flow density $\boldsymbol{j}=0$, the vector potential $\boldsymbol{A}=0$ and the scalar potential degenerates into Newton's gravitational potential $\phi(r)=-\frac{G \rho}{r}$ when the source material is in the static spherical symmetric distribution.

If we define $\eta \equiv G / c^{2}$, the equations (15) and (16) can be written into one equation

$$
A_{\mu}(x)=-\eta \frac{j_{\mu}\left(x^{\prime}\right)}{r} .
$$

Since $\eta=G / c^{2}=7.41 \times 10^{-28} \mathrm{~N} \cdot \mathrm{kg}^{2} \cdot \mathrm{s}^{2}$ is very small, it's very difficult to observe the gravitational waves in usual experiments.

Make a sphere of infinitesimal radius $\mathcal{E}$ at the center $\vec{x}^{\prime}=0$ and integral over the sphere, there is 


$$
\int_{0}^{\varepsilon} d V\left(\nabla^{2}-\frac{1}{c^{2}} \partial_{t}^{2}\right) A_{\mu}(x)=4 \pi \eta j_{\mu}\left(x^{\prime}\right) .
$$

This is because of the second term $\sim \mathcal{E}^{2}$ approaches to zero when $\varepsilon \rightarrow 0$ in the above equation, and $\nabla^{2} \frac{1}{r}=-4 \pi \delta(r)$. So, we can write

$$
\square A_{\mu}(x)=\partial_{v}^{2} A_{\mu}(x)=4 \pi \eta j_{\mu}\left(x^{\prime}\right) \delta(r) .
$$

This is the motion equation of gravitational field.

The right side of the equation above equals zero out of the field source, the motion equation of the free gravitational field is

$$
A_{\mu}(x)=\partial_{v}^{2} A_{\mu}(x)=0
$$

This is a linear homogeneous wave equation, its plane wave solution is

$$
A_{\mu}(x)=e_{\mu} e^{i k x},
$$

where $e_{\mu}$ is the polarization vector of gravitational wave. As shown in the equation (10), the gravitational potential $A_{\mu}$ satisfies the Lorentz condition:

$$
\partial_{\mu} A_{\mu}(x)=0
$$

then there is

$$
k_{\mu} e_{\mu}=0
$$

This shows the gravitational wave is the transverse wave, and it can be proved that only $e_{1}, e_{2}$ are not zero with the method like the theory of electromagnetic field (Weinberg, 1972).

Substitute the equation (21) into (20), we have

$$
k^{2}=k_{v} k_{v}=0
$$

This shows the mass of graviton equals zero, and the velocity of gravitational wave equals the light velocity in the vacuum. So, the gravitational field is a vector field without mass, namely a gauge field. This conclusion is consistent with Utiyama's findings (Utyiama, 1956).

We know that if the transformation equation for the rotation angle $\theta$ of the plane wave around the propagation direction is

$$
\psi^{\prime}=e^{i h \theta} \psi,
$$

we say its helicity is $h$ (Weinberg, 1972). Now assume the rotation angle of the polarization vector of plane gravitational wave around the propagation direction is $\theta$, the transformation equation is

$$
e_{i}^{\prime}=R_{i}^{j} e_{j},
$$

where the rotation matrix is

$$
\left(R_{i}^{j}\right)=\left(\begin{array}{ccc}
\cos \theta & \sin \theta & 0 \\
-\sin \theta & \cos \theta & 0 \\
0 & 0 & 1
\end{array}\right) .
$$

Take $\psi_{ \pm}=e_{ \pm}=e_{1} \mp i e_{2}$, it can be proved that the spin of graviton equals one (the prove process is omitted here). 
In some literature the spin of graviton equals 2, and this is because the polarization tensor $e_{\mu v}$ of plane gravitational wave rotates artificially twice around the wave propagation direction (Weinberg, 1972):

$$
e_{\mu v}^{\prime}=R_{\mu}^{\rho} R_{v}^{\sigma} e_{\rho \sigma}
$$

\section{The Canonical Quantization of Gravitational Field}

We need to know the Lagrange density $L\left(A_{\mu}, \partial_{v} A_{\mu}\right)$ for the quantization of gravitational field. Since the graviton is boson, the gravitational field is an Abel gauge field, and its Lagrange density with gauge invariance is

$$
L=-\frac{1}{4} F_{\mu v} F_{\mu \nu}=-\frac{1}{4}\left(\partial_{v} A_{\mu}-\partial_{\mu} A_{v}\right)^{2},
$$

where $F_{\mu \nu}=\partial_{\nu} A_{\mu}-\partial_{\mu} A_{v}$ is the intensity of gravitational field. Under the Lorentz condition the equation above is equivalent to the equation as follows:

$$
L=-\frac{1}{2}\left(\frac{\partial A_{\mu}}{\partial x_{v}}\right)^{2} .
$$

Modeling on the method of QED, the energy-momentum tensor of the free gravitational field could be obtained from the equation above:

$$
T_{\mu \nu}=\frac{\partial A_{\lambda}}{\partial x_{\mu}} \cdot \frac{\partial A_{\lambda}}{\partial x_{v}}-\frac{1}{2}\left(\frac{\partial A_{\lambda}}{\partial x_{\rho}}\right)^{2} \delta_{\mu \nu},
$$

and the angular momentum density is

$$
M_{\mu v, \lambda}=x_{v} T_{\mu \lambda}-x_{\mu} T_{v \lambda}+\frac{\partial A_{v}}{\partial x_{\lambda}} A_{\mu}-\frac{\partial A_{\mu}}{\partial x_{\lambda}} A_{v} .
$$

Since the gravitational potential $A_{\mu}$ is 4 -vector to satisfy the Lorentz transformation, its spin

$$
S_{\mu \nu ; \sigma \rho}=\delta_{\sigma \mu} \delta_{\rho \nu}-\delta_{\sigma \nu} \delta_{\rho \mu},
$$

The spin of graviton equals one that can be known from the equation above, and this conclusion is consistent with the previous conclusion.

Take $c=\hbar=1$ in the following discussion.

Introduce the conjugate "canonical momentum" $\pi_{\mu}$ for the potential $A_{\mu}$ :

$$
\pi_{\mu}=\frac{\partial L}{\partial \dot{A}_{\mu}}=\dot{A}_{\mu},
$$

where $\dot{A}_{\mu}=\partial_{t} A_{\mu}$. Thus the motion equation (20) is rewritten into the canonical form:

$$
\frac{\partial A_{\mu}}{\partial t}=\pi_{\mu}, \quad \frac{\partial \pi_{\mu}}{\partial t}=\nabla^{2} A_{\mu} .
$$

Hamilton density is

$$
H=\pi_{\mu} \dot{A}_{\mu}-L=\frac{1}{2}\left(\nabla A_{\mu} \cdot \nabla A_{\mu}+\pi_{\mu} \pi_{\mu}\right)
$$

For $t^{\prime}=t$ take

$$
\left[A_{\mu}(x), \pi_{v}\left(x^{\prime}\right)\right]=i \delta_{\mu \nu} \delta\left(\vec{x}-\vec{x}^{\prime}\right),
$$

and other physical quantities are commutable to each other. By use of those commutation relations the equation (35) can be rewritten as 


$$
\frac{\partial A_{\mu}}{\partial t}=i\left[H, A_{\mu}\right], \quad \frac{\partial \pi_{\mu}}{\partial t}=i\left[H, \pi_{\mu}\right],
$$

where Hamilton $H=\int d^{4} x H$.The equations (36) (38) are the basic formulae of the gravitational field quantization, where each of dynamical variables is an operator.

As Fermi pointed out in the electromagnetic field quantization, it should be not asked that Lorentz conditions $\partial_{\mu} A_{\mu}=0$ satisfy the equation (37) in the gravitational field quantization. The meaning of Lorentz conditions is that only the wave function to make the average value of $\partial_{\mu} A_{\mu}$ equals zero represents the objective possible state.

To show the particle property of gravitational field, namely the existence of gravitons, rewrite the free gravitational field into the superposition of plane waves:

$$
A_{\mu}(x)=\sum_{\boldsymbol{k}} \frac{1}{\sqrt{2 \omega}} e_{\mu \sigma}(\boldsymbol{k})\left[b_{\sigma, \boldsymbol{k}} e^{i k x}+b_{\sigma, k}^{+} e^{-i k x}\right]
$$

where the four unit vectors $e_{\mu \sigma}(\sigma=1,2,3,4)$ are orthogonal to each other:

$$
e_{\mu \sigma} e_{\mu \rho}=\delta_{\sigma \rho}, \quad \sigma, \rho=1,2,3,4
$$

which are only the space components but not the time components for the three unit vectors of $\sigma=1,2,3$, and only the time component but not the space component for the vector of $\sigma=4$, that is

$$
e_{\mu i}=\left\{\begin{array}{l}
e_{j i}=1, \\
e_{4 i}=0,
\end{array} \quad e_{\mu 4}=\left\{\begin{array}{l}
e_{i 4}=0, \\
e_{44}=1 .
\end{array} \quad i=1,2,3\right.\right.
$$

Assume the space components of $e_{\mu \sigma}$ for $\sigma=3$ and $\sigma=1,2$ are parallel and vertical to the wave vector $\boldsymbol{k}$, respectively, the commutation relation of operators $b_{\sigma, k}$ and $b_{\sigma, k}^{+}$is

$$
\left[b_{\sigma, k}, b_{\rho, k^{\prime}}^{+}\right]=\delta_{\sigma \rho} \delta_{k k^{\prime}}
$$

in addition, the $b_{i, \boldsymbol{k}}$ and $b_{i, \boldsymbol{k}}^{+}$are Hermitian conjugate and the $b_{4, \boldsymbol{k}}$ and $b_{4, \boldsymbol{k}}^{+}$are anti-Hermitian conjugate to each other for $\sigma=1,2,3$ :

$$
b_{i, k}^{+}=b_{i, k}^{*}, \quad b_{4, k}^{+}=-b_{4, k} .
$$

Thus Hamilton of the gravitational field can be written as

$$
H=\sum_{k} \sum_{\sigma=1}^{4}\left(N_{\sigma, k}+\frac{1}{2}\right) \omega,
$$

and the total momentum is

$$
\boldsymbol{P}=\sum_{\boldsymbol{k}} \sum_{\sigma=1}^{4} N_{\sigma, \boldsymbol{k}} \boldsymbol{k}
$$

where $N_{\sigma, k}=b_{\sigma, k}^{+} b_{\sigma, k}$ is the particle number as operator, and the gravitons for $\sigma=1,2$ are called the transverse gravitons, those for $\sigma=3$ are called the longitudinal gravitons, and those for $\sigma=4$ are called the scalar gravitons. Modeling on the electromagnetic field quantization we can prove that the spin of transverse and longitudinal gravitons is equal to 1 and that of scalar gravitons is equal to zero. The physical effect of longitudinal and scalar gravitons results in the static gravitational field and only transverse gravitons could be observed in the experiments due to the Lorentz condition. There $b_{\sigma, k}^{+}$is the creation operator and $b_{\sigma, k}$ is the annihilation operator of gravitons after the introduction of variable metric. 
It is worth noting that the commutation relation (37) does not have the obvious Lorentz invariance. To get the whole theory with the obvious Lorentz invariance, from equations (35) and (38) we obtain

$$
\left[A_{\mu}(x), A_{v}\left(x^{\prime}\right)\right]=i \delta_{\mu \nu} D\left(\boldsymbol{x}-\boldsymbol{x}^{\prime}\right),
$$

the equation above has the obvious Lorentz invariance, where

$$
D(x)=\frac{-i}{(2 \pi)^{3}} \int d^{4} k e^{i k x} \delta\left(k^{2}\right) \varepsilon\left(k_{0}\right),
$$

and $\varepsilon\left(k_{0}\right)=k_{0} /\left|k_{0}\right|$.

\section{The Renormalization of Gravitational Field}

The gravitational field as one of gauge fields should be renormalizable (Hooft \& Veltman, 1972). This can be proved as follows.

If we take the dimension of mass is 1 and the dimension of Lagrange density is 4 , then the dimension of boson and fermion is 1 and 3/2, respectively. According to the renormalization theory of gauge fields (Dyson, 1949; Zimmermann, 1970), if the behavior of propagator is normal for great momentum and the dimension $d_{v}=4-d_{g} \leq 4$ of field operator product in the interactional Lagrange density $\mathrm{L}_{\mathrm{i}}$, or the dimension $d_{\mathrm{g}} \geq 0$ of the coupling constant, then the theory is renormalizable. Because of that the number of external line satisfies the condition ( $E_{B}$ and $E_{F}$ is the number of boson and fermion external lines):

$$
E_{B}+\frac{3}{2} E_{F} \leq d_{v}
$$

namely only limited several vertexes are divergence in the theory, they can be eliminated by the introduction of limited several elimination terms. For example, the dimension of coupling constant of electromagnetic field or non-Abel gauge theory equals zero, the two theories can be renormalizable. But the dimension $d_{W}=-2<0$ of coupling constant of Fermi weak interaction theory, it is non-renormalizable.

From the equation (20) of gravitational field the propagator can be obtained:

$$
S(k)=-\frac{i}{k^{2}+i \varepsilon}
$$

This shows that the behavior of the propagator is normal for great momentum. What is the dimension $d_{g}$ of the coupling constant of gravity then?

We know that Lagrange density of neutral free bosons (Bjorken \& Drell, 1965) is

$$
L_{0}=-\frac{1}{2}\left[\left(\partial_{\mu} \varphi\right)^{2}+m^{2} \varphi^{2}\right] .
$$

Without the loss of generality, assume the coupling of particle and gravity has nothing with electric charge. Use the covariant differentiation $D_{\mu} \equiv \partial_{\mu}-i g A_{\mu}$ instead of the partial derivative of the equation above, and the Lagrange density of the system of neutral bosons with the gravitational field is obtained

$$
L=-\frac{1}{2}\left[\left(\partial_{\mu} \varphi-i g A_{\mu} \varphi\right)^{2}+m^{2} \varphi^{2}\right]-\frac{1}{2}\left(\frac{\partial A_{\sigma}}{\partial x_{v}}\right)^{2},
$$

where $A_{\mu}$ is the potential of gravity, and the interactional Lagrange density is

$$
L_{i}=i g \varphi A_{\mu} \partial_{\mu} \varphi+\frac{1}{2} g^{2} A_{\mu}^{2} \varphi^{2} .
$$

Because the dimension of $A_{\mu}$ equals 1 , then $d_{g}=0$.

Again for instance, the Lagrange density of free fermions (Bjorken \& Drell, 1965) is 


$$
L_{0}=-\bar{\psi} \gamma_{\mu} \partial_{\mu} \psi-m \bar{\psi} \psi,
$$

and the Lagrange density of the system of fermions with the gravitational field is

$$
L=-\bar{\psi} \gamma_{\mu}\left(\partial_{\mu}-i g A_{\mu}\right) \psi-m \bar{\psi} \psi-\frac{1}{2}\left(\frac{\partial A_{\sigma}}{\partial x_{v}}\right)^{2},
$$

where the interactional Lagrange density is

$$
L_{i}=i g \bar{\psi} \gamma_{\mu} A_{\mu} \psi
$$

Then there is $d_{g}=0$, too. Therefore, the quantum gravitational field is renormalizable.

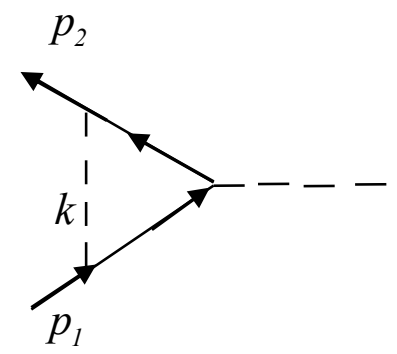

Figure 1. Vertex

The renormalization of the quantum field can be carried out by different methods. The basic idea is to separate the divergent terms from the integral and eliminate them by the elimination terms. In the case of the equation (55), there is only the vertex of Fermion external number $E_{F}=2$ which has the divergence in the interaction, as shown in Figure 1. According to the Feynman rules in quantum field theory, the vertex function can be written as following (Bjorken \& Drell, 1965):

$$
\Lambda_{\mu}\left(p_{1}, p_{2}\right)=\frac{i g^{2}}{(2 \pi)^{4}} \int \frac{\mathrm{d}^{4} k}{k^{2}} \gamma_{\nu} \frac{i\left(\hat{p}_{2}-\hat{k}\right)-m}{\left(p_{2}-k\right)^{2}+m^{2}} \gamma_{\mu} \frac{i\left(\hat{p}_{1}-\hat{k}\right)-m}{\left(p_{1}-k\right)^{2}+m^{2}} \gamma_{\nu} .
$$

In order to keep the internal symmetry of the theory, including the gauge invariance and Lorentz invariance, we calculate the equation above by the dimensional regularization method (Bjorken \& Drell, 1965). The basic idea of the method is to transform the original integral from the 4-momentum space into the $n$-momentum space in order to make the original divergent integral the convergent integral, and then take $n \rightarrow 4$ after the integral, thus the divergent term has been separated.

The conventional calculation rules of $\gamma$ matrix in the dimensional regularization method are as follows:

$$
\begin{array}{cc}
\left\{\gamma_{\mu}, \gamma_{v}\right\}=2 \delta_{\mu \nu}, & \operatorname{Tr}\left(\gamma_{\mu} \gamma_{v}\right)=4 \delta_{\mu \nu}, \quad \operatorname{Tr}(\text { odd } \gamma)=0, \\
\gamma_{\mu} \gamma_{\mu}=4, & \gamma_{\mu} \gamma_{\nu} \gamma_{\mu}=-2 \gamma_{v}, \quad \operatorname{Tr}(I)=4 .
\end{array}
$$

The vertex function in the $n$-momentum space is

$$
\Lambda_{\mu}\left(p_{1}, p_{2}\right)=\frac{i g^{2} \mu^{(4-n)}}{(2 \pi)^{n}} \int \frac{\mathrm{d}^{n} k}{k^{2}} \gamma_{v} \frac{i\left(\hat{p}_{2}-\hat{k}\right)-m}{\left(p_{2}-k\right)^{2}+m^{2}} \gamma_{\mu} \frac{i\left(\hat{p}_{1}-\hat{k}\right)-m}{\left(p_{1}-k\right)^{2}+m^{2}} \gamma_{v},
$$

where the factor $\mu^{(4-n)}$ is introduced in order to keep the dimension of $\Lambda_{\mu}(p)$ invariable. Using the Feynman parameters formula 


$$
\frac{1}{a_{1} a_{2} \cdots a_{n}}=(n-1) ! \int_{0}^{1} d x_{1} \int_{0}^{x_{1}} d x_{2} \cdots \int_{0}^{x_{n-2}} d x_{n-1} \frac{1}{f^{n}},
$$

where

$$
f=a_{1} x_{n-1}+a_{2}\left(x_{n-2}-x_{n-1}\right)+\cdots a_{n}\left(1-x_{1}\right),
$$

and $p_{2}=p_{1}+k_{1}$ (see Figure 1), then the equation (58) can be simplified as

$$
\begin{aligned}
\Lambda_{\mu}\left(p_{1}, p_{2}\right)= & i 4 g^{2} \mu^{(4-n)} \\
& \times \int \frac{\mathrm{d}^{n} k}{(2 \pi)^{n}} \int_{0}^{1} d x \int_{0}^{x} d y \frac{\left(\hat{p}_{1}-\hat{k}\right) \gamma_{\mu}\left(\hat{p}_{2}-\hat{k}\right)-i 2 m\left(p_{1}+p_{2}-2 k\right)_{\mu}-m^{2}}{\left[\left(k-p_{1} x-k_{1} y\right)^{2}+l^{2}\right]^{3}},
\end{aligned}
$$

where

$$
l^{2}=\left(m^{2}+p_{1}^{2}\right) x+k_{1}\left(p_{1}+p_{2}\right) y-\left(k_{1} y+p_{1} x\right)^{2} .
$$

The equation (60) is a convergent integral, and its variables can be transformed. Take $k-p_{1} x-k_{1} y \rightarrow k$, we obtain

$$
\Lambda_{\mu}\left(p_{1}, p_{2}\right)=i 4 g^{2} \mu^{(4-n)} \int \frac{\mathrm{d}^{n} k}{(2 \pi)^{n}} \int_{0}^{1} d x \int_{0}^{x} d y \frac{T}{\left[k^{2}+l^{2}\right]^{3}}
$$

where

$$
\begin{gathered}
T=\left(\hat{p}_{1}(1-x)-\hat{k}_{1} y-\hat{k}\right) \gamma_{\mu}\left(\hat{p}_{2}-\hat{p}_{1} x-\hat{k}_{1} y-\hat{k}\right)-m^{2} \gamma_{\mu} \\
-i 2 m\left(p_{1 \mu}+p_{2 \mu}-2 p_{1 \mu} x-2 k_{1 \mu} y-2 k_{\mu}\right) .
\end{gathered}
$$

The integral result of the odd terms equals zero, and the contribution of each term of $k_{1}^{2}, k_{2}^{2}, k_{3}^{2}, k_{4}^{2}$ for the integral equals the contribution of $k^{2} / 4$ in the equation (61) due to the integral symmetry. Notice $\gamma_{\nu} \gamma_{\mu} \gamma_{v}=-2 \gamma_{\mu}$, the $T$ can be written as

$$
\begin{gathered}
T=\left(\hat{p}_{1}(1-x)-\hat{k}_{1} y-\hat{k}\right) \gamma_{\mu}\left(\hat{p}_{2}-\hat{p}_{1} x-\hat{k}_{1} y-\hat{k}\right)-m^{2} \gamma_{\mu} \\
-\frac{1}{2} k^{2} \gamma_{\mu}-i 2 m\left(p_{1 \mu}+p_{2 \mu}-2 p_{1 \mu} x-2 k_{1 \mu} y\right) .
\end{gathered}
$$

For simplicity, take approximately

$$
p_{2}=p_{1}=p, \quad k_{1}=p_{2}-p_{1}=0,
$$

So the $T$ is simplified as

$$
T=(1-x)^{2} \hat{p} \gamma_{\mu} \hat{p}-\frac{1}{2} k^{2} \gamma_{\mu}-m^{2} \gamma_{\mu}-i 2 m(1-x)\left\{\gamma_{\mu}, \hat{p}\right\}
$$

Since $p^{2}=-m^{2}$, take $i m$ instead of $\hat{p}$, thus there is 


$$
T=\gamma_{\mu}\left\{m^{2}\left(2-2 x-\frac{1}{2} x^{2}\right)-\frac{1}{2}\left(k^{2}+m^{2} x^{2}\right)\right\} .
$$

At the same time, the $l^{2}$ of equation (60) is simplified as

$$
l^{2}=m^{2} x^{2} .
$$

Substitute the two equations above into the equation (61) we obtain

$$
\Lambda_{\mu}\left(p_{1}, p_{2}\right)=i 4 g^{2} \mu^{(4-n)} \gamma_{\mu} A,
$$

where

$$
A=\int \frac{\mathrm{d}^{n} k}{(2 \pi)^{n}} \int_{0}^{1} d x \cdot x\left\{\frac{m^{2}\left(4-4 x-x^{2}\right)}{\left(k^{2}+m^{2} x^{2}\right)^{3}}-\frac{1}{\left(k^{2}+m^{2} x^{2}\right)^{2}}\right\}
$$

The integral space over $k$ in the equation above is a complex Minkowski space, $k^{2}=k_{1}^{2}+\cdots+k_{n-1}^{2}-k_{0}^{2}$, the volume element $d^{n} k=d k_{1} \cdots d k_{n-1} d k_{n}=i d k_{1} \cdots d k_{n-1} d k_{0}$, and the integral function is not analytic. In order to make the integral function analytic, separate the imaginary symbol from the complex volume element $d^{n} k$ : $d^{n} k=i d^{n} k_{E}$, where $d^{n} k_{E}=|k|^{n-1} d|k| d \Omega^{n}$ is the $n$-real volume element(Euclid space). Thus the integral has been transformed into the $n$-real space, $k^{2}=k_{1}^{2}+\cdots+k_{n-1}^{2}+k_{0}^{2}$, and the integral function becomes analytic. Integrate the second term of the equation (63), there is

$$
\int_{0}^{1} \frac{x d x}{\left(k^{2}+m^{2} x^{2}\right)^{2}}=\frac{1}{2} \frac{1}{\left(k^{2}+m^{2} x^{2}\right)^{2}}+\int_{0}^{1} \frac{2 m^{2} x^{3} d x}{\left(k^{2}+m^{2} x^{2}\right)^{3}} .
$$

Substitute the equation above into the equation (63), and by use of the formula

$$
\int \frac{\mathrm{d}^{n} k_{E}}{(2 \pi)^{n}} \frac{1}{\left[k^{2}+l^{2}\right]^{\alpha}}=\frac{\left(l^{2}\right)^{\frac{n}{2}-2}}{(4 \pi)^{n / 2}} \frac{\Gamma\left(\alpha-\frac{n}{2}\right)}{\Gamma(\alpha)},
$$

we obtain

$$
A=-\frac{i}{2}\left(1+\frac{1}{12} m^{2}\left(\frac{4-n}{2}\right)\right) \frac{\left(m^{2} x^{2}\right)^{\frac{n}{2}-2}}{(4 \pi)^{n / 2}} \Gamma\left(\frac{4-n}{2}\right) .
$$

Substitute the equation above into the equation (60), when $n \rightarrow 4$ we have

$$
\Lambda_{\mu}\left(p_{1}, p_{2}\right)=\frac{g^{2}}{8 \pi^{2}} \gamma_{\mu}\left(\frac{1}{\varepsilon}-\gamma_{E}+\cdots\right)
$$

where the Euler's constant $\gamma_{E}=0.57721 \cdots$. The first term in the equation above is divergent when $\varepsilon \rightarrow 0$. So,

the renormalization has been realized by adding the elimination term $-\frac{g^{2}}{8 \pi^{2}} \frac{\gamma_{\mu}}{\varepsilon}$ into the equation (55). 


\section{Reference}

Bjorken, J. D., \& Drell, S. D. (1965). Relativistic Quantum Fields. McGraw-Hill Book Company.

Dyson, F. J. (1949). The S matrix in quantum electrodynamics. Physical Review, 75(11), 1736.

Einstein, A. (1916). Die grundlage der allgemeinen relativitätstheorie. Annalen der Physik, 354(7), 769-822.

Hooft, G., \& Veltman, M. (1972). Nucl Phys., B44, 189. Diagram. CERN Theoretical Studies Division, 1973.

Utyiama, T. (1956). Invariant Theoretical Interpretation of Interactions. Phys. Rev, 101, 1597.

Weinberg, S. (1972). Gravitation and cosmology: principles and applications of the general theory of relativity (Vol. 1). New York: Wiley.

Yang, C. N., \& Mills, R. L. (1954). Conservation of isotopic spin and isotopic gauge invariance. Physical review, 96(1), 191.

Zhu, H. Y. (1960). The theory of Quantum Fields (pp. 66-78). Beijing: Science Press. (in Chinese)

Zimmermann, W. (1970). Lectures on elementary particles and quantum field theory. In S. Deser, M. Grisaru, \& H. Pendleton (Eds.), Brandeis Summer Institute, 1. Cambridge: MIT Press.

\section{Copyrights}

Copyright for this article is retained by the author(s), with first publication rights granted to the journal.

This is an open-access article distributed under the terms and conditions of the Creative Commons Attribution license (http://creativecommons.org/licenses/by/3.0/). 